\title{
Theoretical Studies on the Local Structure and Spin Hamiltonian Parameters for Single $\mathrm{Cu}^{2+}$ Ion in $\mathrm{BaF}_{2}$
}

\author{
J.Z. Lin*, P. XiaO, Y. ZhaO, B.F. Zhang AND Y. YANG \\ Department of Physics, Suzhou Institute of Technology, Jiangsu University of Science and Technology, \\ Zhangjiagang, 215600, P.R. China
}

(Received August 2, 2015; revised version March 21, 2016; in final form April 4, 2016)

The spin Hamiltonian parameters ( $g$-factors and the hyperfine structure constants) and local structure are theoretically studied for single $\mathrm{Cu}^{2+}$ ion in $\mathrm{BaF}_{2}$ from the high-order perturbation formulae of these parameters for $3 \mathrm{~d}^{9}$ ions in tetragonally elongated octahedra. In the calculations, the ligand orbital and spin-orbit coupling of the impurity $\mathrm{Cu}^{2+}$ are taken into account, based on the cluster approach. Due to the Jahn-Teller effect and size mismatching substitution, the impurity $\mathrm{Cu}^{2+}$ is found to be located at a distance of about $0.2 \AA$ from the nearest fluorine plane. The signs of the hyperfine structure constants $A_{\|}$and $A_{\perp}$ are suggested. The theoretical spin Hamiltonian parameters based on the above local structure are in good agreement with the observed values.

DOI: 10.12693/APhysPolA.129.1173

PACS/topics: 75.10.Dg, 76.30.Fc

\section{Introduction}

Fluorite-type crystals $\mathrm{MX}_{2}(\mathrm{M}=\mathrm{Sr}, \mathrm{Ba}, \mathrm{Ca}, \mathrm{Cd}$, $\mathrm{X}=\mathrm{F}, \mathrm{Cl}$ ) are of simple cubic structures, and the divalent cation $\mathrm{M}^{2+}$ is coordinated by eight $\mathrm{X}^{-}$anions which locate at the corners of the unit cell. Many paramagnetic ions (such as $\mathrm{Cu}^{2+}[1-4], \mathrm{Ag}^{2+}[5], \mathrm{Ni}^{+}[6,7], \mathrm{Cr}^{2+}[8]$, $\left.\mathrm{Mn}^{2+}[9], \mathrm{Fe}^{3+}[10,11], \mathrm{Ce}^{3+}[12], \mathrm{Ti}^{3+}[13]\right)$ have been introduced into cubic fluorite-type crystals to study the local structure of the host lattice and the electronic properties of paramagnetic impurities in these crystals by means of electron paramagnetic resonance (EPR) technique, which would be useful to understand the properties (such as optical and luminescent properties) of crystals containing ions. Based on the EPR experiments and theoretical studies, it is found that the local structures around many paramagnetic centers in these crystals are of tetragonal symmetry or orthorhombic symmetry [8]. For example, Hoffmann et al. have studied the EPR spectra of individual $\mathrm{Cu}^{2+}$ ion in $\mathrm{BaF}_{2}$ crystal and got the spin Hamiltonian (SH) parameters $\left(g_{\|}, g_{\perp}, A_{\|}, A_{\perp}\right)$ [3]. Based on the EPR results, it is found that the impurity $\mathrm{Cu}^{2+}$ ions replacing the central cations do not occupy exactly the host cation site but have a large offcentre shift along $\mathrm{C}_{4}$ axis [3]. As a result, the impurity $\mathrm{Cu}^{2+}$ would be much more close to the fluorine plane in the cube and the near square planar $\left[\mathrm{CuF}_{4}\right]^{2-}$ cluster (i.e., the $\mathrm{Cu}^{2+}$ has a small distance $\Delta Z$ from the plane) is formed. (Note: similar situations are also found in other fluorine-type crystals doped with paramagnetic ions, e.g. $\mathrm{Cu}^{2+}$ in $\mathrm{SrCl}_{2}$ and $\mathrm{SrF}_{2}[1,2,4], \mathrm{Ni}^{+}$in $\mathrm{CaF}_{2}$ and $\mathrm{SrF}_{2}[6,7]$.) Till date, however, the above experimental results have not been satisfactorily interpreted.

*corresponding author; e-mail: jizi_lin@hotmail.com
For instance, the SH parameters were not theoretically explained, and the relationship between the SH parameters and the local structure is not clarified. Since the microscopic mechanisms of the EPR spectra and information about local defect structures would be useful to understand the properties of these materials containing transition ions, theoretical studies on the $\mathrm{SH}$ parameters and the local structures of $\mathrm{Cu}^{2+}$ ions in $\mathrm{BaF}_{2}$ crystals are of fundamental and practical significance.

In present studies, the high-order perturbation formulae of the $\mathrm{SH}$ parameters for $3 d^{9}$ ions under tetragonally elongated octahedra are adopted for analysis of the above mentioned $\mathrm{Cu}^{2+}$ center. In these formulae, the contributions from the ligand orbital and spin-orbit coupling interactions are taken into account from the cluster approach and the energy denominators are correlated with the local structure around the impurity $\mathrm{Cu}^{2+}$ center and hence the local structure of $\mathrm{Cu}^{2+}$ in $\mathrm{BaF}_{2}$ can be quantitatively determined on the basis of the EPR analysis. The results are discussed.

\section{Calculations}

For $\mathrm{Cu}^{2+}\left(3 d^{9}\right)$ ions in tetragonally elongated octahedra, the lower ${ }^{2} E$ irreducible representation may be separated into the orbital singlets ${ }^{2} B_{1}\left(\left|x^{2}-y^{2}\right\rangle\right)$ and ${ }^{2} A_{1}\left(\left|z^{2}\right\rangle\right)$, with the former lying lowest, while the upper ${ }^{2} T_{2}$ representation would split into an orbital singlet ${ }^{2} B_{2}(|x y\rangle)$ and a doublet ${ }^{2} E(|x z\rangle,|y z\rangle)$ [14]. In an octahedral cluster, the total single electron wave functions including the contributions from $p$ - and $s$-orbitals of ligands may be written as $[15,16]$ :

$$
\begin{aligned}
& \psi_{t}=N_{t}^{1 / 2}\left(\phi_{t}-\lambda_{t} \chi_{p t}\right), \\
& \psi_{e}=N_{e}^{1 / 2}\left(\phi_{e}-\lambda_{e} \chi_{p e}-\lambda_{s} \chi_{s}\right) .
\end{aligned}
$$

Here, the subscript $\gamma(=t$ and $e$ ) denotes the irreducible representation of $O_{h}$ group. $\phi_{\gamma}$ is the $d$-orbital of the $3 d^{n}$ 
ion. $\chi_{p \gamma}$ and $\chi_{s}$ are the $p$-orbital and $s$-orbital of ligand. $N_{\gamma}$ denotes the normalization factors and $\lambda_{\gamma}\left(\right.$ or $\left.\lambda_{s}\right)$ are the orbital mixing coefficients, they are determined from the approximate relationships $[16,17]$ :

$$
\begin{aligned}
& N^{2}=N_{t}^{2}\left[1+\lambda_{t}^{2} S_{d p t}^{2}-2 \lambda_{t} S_{d p t}\right] \\
& N^{2}=N_{e}^{2}\left[1+\lambda_{e}^{2} S_{d p e}^{2}+\lambda_{s}^{2} S_{d s}^{2}-2 \lambda_{e} S_{d p e}-2 \lambda_{s} S_{d s}\right]
\end{aligned}
$$
and the normalization conditions $[16,17]$ :

$$
\begin{aligned}
& N_{t}\left(1-2 \lambda_{t} S_{d p t}+\lambda_{t}^{2}\right)=1, \\
& N_{e}\left(1-2 \lambda_{e} S_{d p e}-2 \lambda_{s} S_{d s}+\lambda_{e}^{2}+\lambda_{s}^{2}\right)=1 .
\end{aligned}
$$

Here, $S_{d p \gamma}$ (and $S_{d s}$ ) are the group overlap integrals. $N$ is the average covalency factor, characteristic of the covalency effect (or reduction of the spin-orbit coupling coefficient and the dipolar hyperfine structure parameter) of the central ion in crystals. The orbital mixing coefficients increase with increase of the group overlap integrals, and one can approximately adopt proportionality relationship $\lambda_{e} / S_{d p e} \approx \lambda_{s} / S_{s}$ between the orbital mixing coefficients and the related group overlap integrals within the same irreducible representation $e_{g}[16,17]$. Thus, from Eq. (1), the spin-orbit coupling coefficients and the orbital reduction factors can be derived and given as follows [16, 17]:

$$
\begin{aligned}
& \zeta=N_{t}\left(\zeta_{d}^{0}+\lambda_{t}^{2} \zeta_{p}^{0} / 2\right), \\
& \zeta^{\prime}=\left(N_{t} N_{e}\right)^{1 / 2}\left(\zeta_{d}^{0}-\lambda_{t} \lambda_{e} \zeta_{p}^{0} / 2\right), \\
& k=N_{t}\left(1+\lambda_{t}^{2} / 2\right), \\
& k^{\prime}=\left(N_{t} N_{e}\right)^{1 / 2}\left[1-\lambda_{t}\left(\lambda_{e}+\lambda_{s} A\right) / 2\right] .
\end{aligned}
$$

In the above formulae, $\zeta_{d}^{0}$ and $\zeta_{p}^{0}$ are the spin-orbit coupling coefficients of the free $3 d^{n}$ and ligand ions, respectively. $A$ denotes the integral $R\left\langle n s\left|\frac{\partial}{\partial y}\right| n p_{y}\right\rangle$, where $R$ is the impurity-ligand distance of the studied system.

Based on the perturbation theory and the cluster approach $[18,19]$, the high-order perturbation formulae of the $\mathrm{SH}$ parameters of the ${ }^{2} B_{1}$ ground state for $3 d^{9}$ ions in tetragonally elongated octahedra can be derived and given as follows:

$$
\begin{aligned}
g_{\|} & =g_{s}+8 k^{\prime} \zeta^{\prime} / E_{1}+k \zeta^{\prime 2} / E_{2}^{2}+4 k^{\prime} \zeta^{\prime} \zeta / E_{1} E_{2} \\
& +g_{e} \zeta^{\prime 2}\left(1 / E_{1}^{2}-1 / 2 E_{2}^{2}\right)-k \zeta \zeta^{\prime 2}\left(4 / E_{1}-1 / E_{2}\right) / E_{2}^{2} \\
& -2 k^{\prime} \zeta^{\prime} \zeta\left(2 / E_{1} E_{2}-1 / E_{2}^{2}\right) / E_{1} \\
& -g_{e} \zeta \zeta^{2}\left(1 / E_{1} E_{2}^{2}-1 / 2 E_{2}^{3}\right), \\
g_{\perp} & =g_{s}+2 k^{\prime} \zeta^{\prime} / E_{2}-4 k \zeta^{\prime 2} / E_{1} E_{2}+k^{\prime} \zeta \zeta^{\prime}\left(2 / E_{1}\right. \\
& \left.-1 / E_{2}\right) / E_{2}+2 g_{e} \zeta^{\prime 2} / E_{1}^{2}+\zeta \zeta^{\prime}\left(k \zeta^{\prime}-k^{\prime} \zeta\right) / E_{1} E_{2}^{2} \\
& -\zeta \zeta^{\prime}\left(1 / E_{1}-2 / E_{2}\right)\left(2 k \zeta^{\prime} / E_{1}+k^{\prime} \zeta / E_{2}\right) / 2 E_{2} \\
& -g_{e} \zeta \zeta^{\prime 2}\left(1 / E_{1}^{2}-1 / E_{1} E_{2}+1 / E_{2}^{2}\right) / 2 E_{2}, \\
A_{\|} & =P(-\kappa-4 / 7)+P^{\prime}\left[\left(g_{\|}-g_{s}\right)+3\left(g_{\perp}-g_{s}\right) / 7\right], \\
A_{\perp} & =P(-\kappa+2 / 7)+P^{\prime}\left[11\left(g_{\perp}-g_{s}\right) / 14\right] .
\end{aligned}
$$

Here, $g_{s} \approx 2.0023$ is the spin-only value, and $k^{\prime}, k, \zeta$, and $\zeta^{\prime}$ are the orbital reduction factors and the spin-orbit coupling coefficients mentioned in Eq. (4), respectively. $P$ and $P^{\prime}$ are the dipolar hyperfine constants related to the interaction within $t_{2 g}$ states and the interaction between $t_{2 g}$ and $e_{g}$ states. They can be given in terms of the corresponding free-ion values, i.e., $P=N_{t} P_{0}$ and $P^{\prime}=\left(N_{t} N_{e}\right)^{1 / 2} P_{0}$, the free-ion value $P_{0}$ of ${ }^{63} \mathrm{Cu}^{2+}$ is $388 \times 10^{-4} \mathrm{~cm}^{-1}[20] . \quad \kappa$ is the core polarization constant. The denominators $E_{1}$ and $E_{2}$ are the crystal field energy levels, they can be expressed in terms of the tetragonal field parameters $D_{s}$ and $D_{t}$ and the cubic field parameter $D_{q}$ :

$$
\begin{aligned}
& E_{1}=E\left({ }^{2} B_{2}\right)-E\left({ }^{2} B_{1}\right)=10 D_{q}, \\
& E_{2}=E\left({ }^{2} E\right)-E\left({ }^{2} B_{1}\right)=10 D_{q}-3 D_{s}+5 D_{t} .
\end{aligned}
$$

For single $\mathrm{Cu}^{2+}$ ion in $\mathrm{BaF}_{2}$, as mentioned before, the $\mathrm{Cu}^{2+}$ ions do not occupy the host $\mathrm{Ba}^{2+}$ site exactly, but have a large off-centre shift along the $\mathrm{C}_{4}$ axis due to the Jahn-Teller effect and the size mismatching substitution (the ionic radius $(\approx 0.73 \AA[3])$ of $\mathrm{Cu}^{2+}$ is much smaller than that $(\approx 1.56 \AA[3])$ of the replaced $\mathrm{Ba}^{2+}$ ion), as a result, the impurity $\mathrm{Cu}^{2+}$ would be much more close to the fluorine plane in the cube and the near square planar $\left[\mathrm{CuF}_{4}\right]^{2-}$ (the $\mathrm{Cu}^{2+}$ has a small distance $\Delta Z$ from the plane) is formed. The rest four ligands in unit cell are much further from the impurity and their influence may be neglected for simplicity. Thus, the crystal field parameters $D_{q}, D_{s}$ and $D_{t}$ in Eq. (6) can be calculated from the point-charge model [21-23] and the geometrical relationship of the studied impurity centers, and can be determined as follows:

$$
\begin{aligned}
& D_{q}=-e q\left\langle r^{4}\right\rangle /\left(6 R^{\prime 5}\right), \\
& D_{s}=-2 e q\left\langle r^{2}\right\rangle\left(3 \cos ^{2} \alpha-1\right) /\left(7 R^{\prime 3}\right), \\
& D_{t}=-e q\left\langle r^{4}\right\rangle\left[35 \cos ^{4} \alpha-30 \cos ^{2} \alpha+3\right. \\
& \left.\quad-7 \sin ^{4} \alpha\right] /\left(42 R^{\prime 5}\right),
\end{aligned}
$$

where $q(=-e)$ is the effective charge of the fluorine ligand. In view of the admixture (or covalency) between the central ion and the ligand orbitals [21-23], the expectation values $\left\langle r^{2}\right\rangle$ and $\left\langle r^{4}\right\rangle$ of the radial wave function of the $3 d^{n}$ orbital in crystals can be reasonably expressed in terms of the corresponding free-ion values [24] and the average covalency factor $N$ :

$$
\left\langle r^{2}\right\rangle \approx 3.11 N \text { [a.u.], }\left\langle r^{4}\right\rangle \approx 44.80 N \text { [a.u.]. }
$$

$R^{\prime} \approx\left(R_{f c}^{2}+\Delta Z^{2}\right)^{1 / 2}$ represents the $\mathrm{Cu}^{2+}-\mathrm{F}^{-}$bonding length from the distance $\Delta Z$ between the impurity and the fluorine plane, $R_{f c}(\approx 2.192 \AA)$ denotes the distance between the face-center site and the corner of the same fluorine plane and can be obtained from the crystal structure parameter of the host lattice [3]. The $\alpha \approx \operatorname{arcos}\left(\Delta Z / R^{\prime}\right)$ is the bonding angle between the $\mathrm{C}_{4}$ axis and the $\mathrm{Cu}^{2+}-\mathrm{F}^{-}$bond in $\left[\mathrm{CuF}_{4}\right]^{2-}$. From the distance $R \approx 2.685 \AA$ (here $R$ is taken as the metalligand distance in host lattice) [3] and the Slater-type 
SCF functions [25, 26], the group overlap integrals $S_{d p t} \approx$ $0.00031, S_{d p e} \approx 0.00171, S_{d s} \approx 0.00058$ and the integral $A \approx 1.6647$ are obtained. For $\mathrm{Cu}^{2+}$ ion in $\mathrm{SrF}_{2}$ which is isomorphous with $\mathrm{BaF}_{2}$, the average covalency factor $N\left(\approx\left(k_{11}+k_{21}+k_{22}\right) / 3 \approx 0.82\right)$ was obtained based on the theoretical analysis of the spin Hamiltonian parameters including partial ligand orbital contributions to these parameters [27], considering that neglecting the ligand orbital contributions may lead to larger spin-orbit coupling coefficient and the orbital reduction factor (see Eq. (4)) and hence to larger $g$-factors (Eq. (5)), the average covalency factor $N \approx 0.76$ which is little smaller than that $(\approx 0.82)$ for $\mathrm{Cu}^{2+}$ ion in $\mathrm{SrF}_{2}$ can be approximately estimated for present system. Then the molecular orbital coefficients $N_{t} \approx 0.766, N_{e} \approx 0.767, \lambda_{t} \approx 0.553$, $\lambda_{e} \approx 0.524$ and $\lambda_{s} \approx 0.178$ are calculated from Eqs. $(2)$ and $(3)$. The parameters $\zeta \approx 661 \mathrm{~cm}^{-1}, \zeta^{\prime} \approx 611 \mathrm{~cm}^{-1}$, $k \approx 0.883$ and $k^{\prime} \approx 0.594$ can be determined from Eq. (4) and the free-ion values $\zeta_{d}^{0}\left(\mathrm{Cu}^{2+}\right) \approx 829 \mathrm{~cm}^{-1}$ [17] and $\zeta_{p}^{0}\left(\mathrm{~F}^{-}\right) \approx 220 \mathrm{~cm}^{-1}[28]$.

Thus, by using the formulae of $g$ factors and hyperfine structure constants, and fitting the calculated results to the experimental data, we have

$$
\Delta Z \approx 0.2 \AA, \kappa \approx 0.343 .
$$

The corresponding SH parameters are shown in Table I. For comparison, the theoretical results based on the local structure parameter in Eq. (9) but neglecting the ligand contributions (i.e. $\zeta^{\prime}=\zeta=N \zeta_{d}^{0}$ and $k=k^{\prime}=N$ ) are also given in Table I.

\section{TABLE I}

The $g$-factors and hyperfine structure constants (in $10^{-4} \mathrm{~cm}^{-1}$ ) for ${ }^{63} \mathrm{Cu}^{2+}$ in $\mathrm{BaF}_{2}$.

\begin{tabular}{c|c|c|c|c}
\hline \hline & $g_{\|}$ & $g_{\perp}$ & $A_{\|}\left[10^{-4} \mathrm{~cm}^{-1}\right]$ & $A_{\perp}\left[10^{-4} \mathrm{~cm}^{-1}\right]$ \\
\hline cal. $^{a}$ & 2.680 & 2.139 & -52.7 & 14.8 \\
cal. $^{b}$ & 2.511 & 2.105 & -107.4 & 7.1 \\
exp. $^{[3]}$ & 2.511 & 2.092 & 98.3 & 7 \\
\hline
\end{tabular}

Calculation based on the local structure parameter $\Delta Z$, neglecting $^{a}$ and including ${ }^{b}$ the ligand contributions.

\section{Discussion}

From Table I, one can find that the theoretical SH parameters based on the high-order perturbation formulae adopted in this work by considering the local structure parameter $\Delta Z$ as well as the ligand orbitals and spinorbit coupling contributions show good agreement with the experimental values. This suggests that the formulae and the related parameters adopted here can be regarded as reasonable and the EPR spectra of single $\mathrm{Cu}^{2+}$ ion in $\mathrm{BaF}_{2}$ are satisfactorily interpreted.

1. The distance $\Delta Z(\approx 0.2 \AA)$ of the impurity $\mathrm{Cu}^{2+}$ from the fluorine plane based on the analysis of the spin Hamiltonian parameters in this work is consistent with that $(\approx 0.23 \AA)$ based on the Fourier transform (FT)-electron spin echo (ESE) spectrum [3]. Our calculations further confirm that the
$\mathrm{Cu}^{2+}$ ion is displaced out of the centre approaching to the nearest fluorine plane along the $\mathrm{C}_{4}$ axis in the lattice. Thus, the near square planar $\left[\mathrm{CuF}_{4}\right]^{2-}$ is formed. In fact, for other fluorite-type crystals doped with $d^{9}$ ions, the large off-centre shift $(\approx$ $3.10 \AA / 2-\Delta Z \approx 1.35 \AA$ for the studied system) was also found, e.g. $\mathrm{Cu}^{2+}$ ion $\mathrm{SrCl}_{2}(\approx 1.35 \AA)[2], \mathrm{Ni}^{+}$ ion in $\mathrm{CaF}_{2}(\approx 0.99 \AA)$ [6] and $\mathrm{SrF}_{2}(\approx 1.08 \AA)$ [7]. Therefore, the local structure parameter $\Delta Z$ of the impurity $\mathrm{Cu}^{2+}$ center in $\mathrm{BaF}_{2}$ obtained in this work can be regarded as reasonable. Actually, when the host $\mathrm{Ba}^{2+}$ is replaced by the much smaller $\mathrm{Cu}^{2+}$, the impurity may be unstable at the host site and then suffer a large displacement due to the size mismatching substitution. As a result, the impurity $\mathrm{Cu}^{2+}$ is very close to the fluorine plane and this center can be conveniently described as $\left[\mathrm{CuF}_{4}\right]^{2-}$. The above local structure model and formulae are also applied to analyze the EPR results and the local structures for $\mathrm{Ni}^{+}$in $\mathrm{CaF}_{2}$ and $\mathrm{SrF}_{2}$ which may further demonstrate the validity and applicability of the present calculations. According to our calculation, the local structure parameter $\Delta Z$ is about $0.35 \AA$ and $0.36 \AA$ for $\mathrm{Ni}^{+}$in $\mathrm{CaF}_{2}$ and $\mathrm{SrF}_{2}$, respectively, based on the cubic field parameter $D_{q} \approx 400 \mathrm{~cm}^{-1}$ for $\left[\mathrm{NiF}_{4}\right]^{3-}$ (it should be noted that $D_{q} \approx 600 \mathrm{~cm}^{-1}$ was obtained for the octahedral $\left[\mathrm{NiF}_{6}\right]^{5-}$ cluster in Ref. [29]) and the average covalency factor $N\left(\approx 0.774\right.$ and 0.797 for $\mathrm{Ni}^{+}$in $\mathrm{CaF}_{2}$ and $\mathrm{SrF}_{2}$, respectively). The distance $\Delta Z$ for $\left[\mathrm{NiF}_{4}\right]^{3-}$ is consistent with those $(\approx 0.37 \AA[6]$ and $0.365 \AA$ [7] for $\mathrm{Ni}^{+}$in $\mathrm{CaF}_{2}$ and $\mathrm{SrF}_{2}$, respectively) obtained in previous works based on the simple second-order perturbation formulae of $g$-factors for $3 d^{9}$ ion $\left(\mathrm{Ni}^{+}\right)$under tetragonal symmetry and that $\left(\approx 0.33 \AA\right.$ for $\mathrm{Ni}^{+}$in $\left.\mathrm{CaF}_{2}\right)$ obtained in the literature [30] based on the density functional theory (DFT). The above results further prove that the defect structure obtained in this work is valid. In addition, the energy separations $E_{1} \approx 6042 \mathrm{~cm}^{-1}$ and $E_{2} \approx 9134 \mathrm{~cm}^{-1}$ are obtained based on the $\Delta Z$ and the analysis of $g$-factors and one can compare them with those $\left(E_{1} \approx 8600 \mathrm{~cm}^{-1}\right.$ and $E_{2} \approx 9500 \mathrm{~cm}^{-1}$ ) of $\mathrm{Cu}^{2+}$ ion in $\mathrm{SrF}_{2}$ [27]. Considering that the crystal-field strength around the impurity $\mathrm{Cu}^{2+}$ ion may mainly depend upon the distances between the impurity ion and the nearest ligand ions (see Eq. (7), e.g., $D_{q} \propto R^{\prime-5}$ ) and that the metal-ligand distance $\left(R^{\prime} \approx 2.20 \AA\right.$ obtained in this work) for $\mathrm{Cu}^{2+}$ ion in $\mathrm{BaF}_{2}$ is larger than that $\left(R^{\prime} \approx 1.97 \AA[4]\right)$ for $\mathrm{Cu}^{2+}$ ion in $\mathrm{SrF}_{2}$, the energy separations can be understood and also support the validity of the local structure parameter $\Delta Z$. Thus, the relationship between the $\mathrm{SH}$ parameters and the local structure is clarified.

2. The studied system exhibits significant covalency and impurity-ligand orbital admixtures, characteristic of the covalency factor $N(\approx 0.76<1)$ and the 
obvious mixing coefficients $\left(\lambda_{t} \approx 0.553, \lambda_{e} \approx 0.524\right.$, $\left.\lambda_{s} \approx 0.178\right)$ obtained from the cluster approach. Meanwhile, the ratios $\left(\zeta+\zeta^{\prime}\right) / 2 / \zeta_{d}^{0}(\approx 0.77)$ and the average $\left(k+k^{\prime}\right) / 2(\approx 0.74)$ in present work approximately account for the covalency effect. From Table I, one can find that the theoretical SH parameters are not in accordance with the experimental results when the ligand orbital and spin-orbit coupling contributions are ignored. Particularly $g_{\|}$ is larger than the experimental data. In fact, neglecting the ligand orbital contributions may lead to larger spin-orbit coupling coefficient and the orbital reduction factor (see Eq. (4)) and hence to larger $g$-factors (Eq. (5)). Based on the studies, inclusion of the contributions from the spinorbit coupling coefficient and the orbitals of the ligands lead to the variations of about 0.07 and 0.04 for the calculated $g_{\|}$and $g_{\perp}$, respectively. Therefore, for present system, the ligand contributions to SH parameters may not be ignored due to the significant covalency effect, although the ligand spin-orbit coupling coefficient $\left(\approx 220 \mathrm{~cm}^{-1}\right.$ [28]) is smaller than that $\left(\approx 829 \mathrm{~cm}^{-1}[17]\right)$ of the central $\mathrm{Cu}^{2+}$ ion (similar situations are also found in other crystals doped with $\mathrm{Cu}^{2+}$ ions $[31,32]$, e.g. $\mathrm{Cu}^{2+}$ ions in $\mathrm{ZnO}$ [31]).

3. It is difficult to determine the signs of the hyperfine structure constants in EPR experiment. Therefore, although the $A$-values of $\mathrm{Cu}^{2+}$ in $\mathrm{BaF}_{2}$ obtained from EPR experiment are positive [3], they are actually absolute values. Our calculations show that $A_{\|}$is negative while $A_{\perp}$ is positive (see $\mathrm{Cal}^{b}$ in Table I). The signs of $A_{\|}$and $A_{\perp}$ suggested here for tetragonal $\mathrm{Cu}^{2+}$ in $\mathrm{BaF}_{2}$ crystal are also supported by the theoretical results for $\mathrm{Cu}^{2+}$ ions in crystals [17, 20, 33, 34] and can be considered as reasonable. Moreover, the core polarization constant $\kappa(\approx 0.343)$ adopted here in the formulae of $A$ constants is closed to the expectation value 0.3 for $3 d^{n}$ ions in crystals $[20,35]$ and can be regarded as valid.

4. The error analysis of the present treatments can be illustrated as the following points. Firstly, the high-order perturbation formulae of the SH parameters based on the cluster approach may cause some errors. Secondly, the metal-ligand distance in host lattice is adopted in the calculations of the group overlap integrals, this can give rise to certain errors. If the actual metal-ligand bonding length (e.g., $R^{\prime} \approx 2.20 \AA$ obtained in this work) is utilized, the errors for the quantities $\left(k^{\prime}, k, \zeta\right.$ and $\left.\zeta^{\prime}\right)$ are not more than $0.4 \%$. Thirdly, the displacements of the fluorine ligands around the impurity $\mathrm{Cu}^{2+}$ are neglected in the analyses. In fact, these $\mathrm{F}^{-}$ions may shift slightly towards the center of the cube with respect to large off-centre displacement of the impurity $\mathrm{Cu}^{2+}$. However, these shifts in magnitude should be much smaller than the offcentre displacement of the impurity $\mathrm{Cu}^{2+}[4]$. For the sake of simplicity and reduction of number of the adjustable parameters, the errors caused by neglection of the above ligand shifts may be taken as absorbed in the distance $\Delta Z$ and covalency factor $N$ in present studies. Therefore, the distance $\Delta Z(\approx 0.2 \AA)$ obtained in this work can be considered as the effective distance between the impurity and its nearest ligand plane, similar treatments are also adopted in previous work to investigate the local structures of some transition metal ions in crystals (e.g., $\left.\mathrm{KTaO}_{3}: \mathrm{Ni}^{3+}[36]\right)[36,37]$.

\section{Conclusions}

The local structure and the spin Hamiltonian parameters for the single $\mathrm{Cu}^{2+}$ center in $\mathrm{BaF}_{2}$ crystal are theoretically investigated from the high-order perturbation formulae of these parameters including the ligand contributions. It is found that the impurity $\mathrm{Cu}^{2+}$ locates at a distance of about $0.2 \AA$ from the nearest fluorine plane, i.e., the $\left[\mathrm{CuF}_{4}\right]^{2-}$ cluster is expected. The negative sign of $A_{\|}$and positive sign of $A_{\perp}$ are suggested.

\section{Acknowledgments}

This work was supported by the Foundation of the College Natural Science Research Project of Jiangsu Province (Grant No. 15KJD140003), the PhD Startup Fund of Jiangsu University of Science and Technology (Grant No. 120140010) and the Youth Foundation of Zhangjiagang campus of Jiangsu University of Science and Technology (Grant No. 126111432).

\section{References}

[1] H. Bill, Phys. Lett. A 44, 101 (1973).

[2] S.K. Hoffmann, V.A. Ulanov, J. Phys. Condens. Matter 12, 1855 (2000).

[3] S.K. Hoffmann, J. Goslar, S. Lijewski, V.A. Ulanov, J. Chem. Phys. 127, 124705 (2007).

[4] V.A. Ulanov, M. Krupski, S.K. Hoffmann, M.M. Zaripov, J. Phys. Condens. Matter 15, 1081 (2003).

[5] M.V. Eremin, V.A. Ulanov, M.M. Zaripov, Appl. Magn. Reson. 14, 435 (1998).

[6] J.C. Gonzales, H.W. Hartog, R. Alcala, Phys. Rev. B 21, 3826 (1980).

[7] P.J. Alonso, J. Casas-Gonzales, H.W. Hartog, R. Alcala, Phys. Rev. B 27, 2722 (1983).

[8] P.B. Oliete, V.M. Orera, P.J. Alonso, Appl. Magn. Reson. 15, 155 (1998).

[9] S. Lijewski, S.K. Hoffmann, J. Goslar, M. Wencka, V.A. Ulanov, J. Phys. Condens. Matter. 20, 385208 (2008).

[10] E.R. Zhiteitsev, V.A. Ulanov, M.M. Zaripov, Phys. Solid State 49, 845 (2007).

[11] S.V. Nistort, E. Goovaerts, D. Schoemaker, J. Phys. Condens. Matter 6, 2619 (1994).

[12] A. Kiel, W.B. Mims, Phys. Rev. B 6, 34 (1972). 
[13] V.A. Ulanov, E.R. Zhiteitcev, A.G. Varlamov, J. Mol. Struct. 838, 182 (2007).

[14] A. Abragam, B. Bleanely, Electron Paramagnetic Resonance of Transition Ions, Oxford University Press, London 1970.

[15] S. Sugano, Y. Tanabe, H. Kamimura, Multiplets of Transition-Metal Ions in Crystals, Academic Press, New York 1970.

[16] X.Y. Gao, S.Y. Wu, W.H. Wei, W.Z. Yan, Z. Naturforsch. 60a, 145 (2005).

[17] H.M. Zhang, J. Phys. Chem. Solids 73, 846 (2012).

[18] S.Y. Wu, X.Y. Gao, H.N. Dong, J. Magn. Magn. Mater. 301, 67 (2006).

[19] H.M. Zhang, J.H. Duan, W.B. Xiao, X. Wan, J. NonCryst. Solids 425, 173 (2015).

[20] B.R. McGarvey, J. Phys. Chem. 71, 51 (1967).

[21] M.G. Zhao, Chin. Phys. Lett. 20, 2229 (2003).

[22] M.G. Zhao, J. Chem. Phys. 109, 8003 (1998).

[23] M.G. Zhao, Y. Lei, J. Phys. Condens. Matter 9, 529 (1997).

[24] Y.P. Huang, L.J. Wang, W.L. Feng, Radiat. Eff. Defect. S. 164, 183 (2009).

[25] E. Clementi, D.L. Raimondi, J. Chem. Phys. 38, 2686 (1963)

[26] E. Clementi, D.L. Raimondi, W.P. Reinhardt, J. Chem. Phys. 47, 1300 (1967).
[27] V.A. Ulanov, O.A. Anikeenok, M.M. Zaripov, I.I. Fazlizhanov, Phys. Solid State 45, 1906 (2003).

[28] S.Y. Wu, X.Y. Gao, Wei Zi Yan, Spectrochim. Acta A 60, 2531 (2004).

[29] J.A. Aramburu, M. Moreno, M.T. Barriuso, J. Phys. Condens. Matter 4, 9089 (1992).

[30] J.A. Aramburu, P. Fernandez, M.T. Garcia, M. Moreno, Phys. Rev. B 67, 020101 (2003).

[31] G.L. Li, S.Y. Wu, Z.H. Zhang, C.C. Ding, X.F. Hua, Physica B 457, 160 (2015)

[32] C.C. Ding, S.Y. Wu, G.L. Li, Y.Q. Xu, Physica B 459, 129 (2015).

[33] C.Y. Li, H. Ying, Z.X. Mei, Physica B 456, 125 (2015).

[34] H.M. Zhang, S.Y. Wu, Z.H. Zhang, Condens. Matter Phys. 14, 23703 (2011).

[35] J.S. Griffith, The Theory of Transition-Metal Ions, Cambridge University Press, London 1964.

[36] S.Y. Wu, H.N. Dong, W.H. Wei, Z. Naturforsch. A 59a, 203 (2004).

[37] W.C. Zheng, S.Y. Wu, Z. Naturforsch. A 57a, 925 (2004). 